\title{
Unsicherheit medizinischer Entscheidungen: wer sind die Gewinner und die Verlierer?
}

\author{
J. Bircher*
}

* Mitglied der Expertengruppe «Zukunft Medizin Schweiz».

1 McNeil BJ, Hidden barriers to improvement in the quality of care. N Engl J Med 2001; 345:1612-20.

Korrespondenz: Prof. Johannes Bircher Reuelweg 20 CH-3045 Meikirch
Der angehende Arzt muss lernen damit zu leben, dass medizinische Entscheidungen immer unsicher sind. Diese Unsicherheiten haben viele Gründe [1]. Zunächst ist schon die Kommunikation mit dem Patienten ein Wagnis. Im Gespräch müssen subjektive Erlebnisse einerseits in pathophysiologische Denkmuster und andererseits in empathisches Verständnis für die individuelle Lebenssituation übersetzt werden. Wie z.B. weiss ich, wieviel und welche Art von Schmerzen eine Patientin erleidet, und was diese Schmerzen für sie bedeuten? Eine gute Anamnese erfordert nicht nur Fachkompetenz, sondern auch kommunikative Kompetenz und genügend Zeit. Aus diesen unsicheren Erhebungen werden dann die Entscheidungen über die diagnostischen Massnahmen getroffen. Ihre Auswahl ist Ermessenssache, und ihre Resultate sind mit der Unsicherheit über ihre Aussagekraft behaftet. Nicht besser steht es um die Therapie. Kaum eine Behandlung unterliegt nicht dem Risiko einer falschen Bewertung oder einer Nebenwirkung. Weitere Quellen für Unsicherheit liegen im wissenschaftlichen Fortschritt und in der Information darüber. Was heute richtig war, ist morgen vielleicht falsch. Wie kann der einzelne Arzt sein Wissen auf der Höhe der aktuellen Entwicklung der Wissenschaft halten?

Trotz all dieser Unsicherheiten müssen Ärztinnen handeln, denn die Patienten konfrontieren sie mit ihren gesundheitlichen Problemen und wollen betreut sein. Verantwortungsbewusste Ärzte haben deshalb schon immer Strategien entwickelt, um mit ihrer Unsicherheit umzugehen. Sie überprüfen die Behandlungsresultate ihrer Patientinnen und unternehmen einen grossen Aufwand für ihre Weiterbildung. Die Ärzteschaft als Ganzes trägt ihrerseits zur Verminderung der Unsicherheit bei, so z.B. durch Förderung der klinischen Forschung an den Universitätskliniken, die Etablierung der Evidencebased Medicine (EBM) und die Erarbeitung von Richtlinien. Trotz vielseitiger Bemühungen bleibt jedoch der grössere Teil der ärztlichen Entscheidungen nicht wissenschaftlich begründbar und damit unsicher. Diese Entscheidungen werden deshalb aufgrund einer etablierten Praxis oder einfach entsprechend dem persönlichen Ermessen der Ärztin gefällt. In jedem Fall hätten sie ebensogut anders getroffen werden können, wobei auch unsicher bleibt, ob dann der Nutzen für den Patienten grösser oder kleiner gewesen wäre. Weil es in der Natur der Medizin liegt, dass für alle Massnahmen mögliche Alternativen existieren, müssen die Ärzte die Restunsicherheit akzeptieren, und mit ihr leben lernen. Deshalb wird sie bei wichtigen Entscheidungen auch den Patienten mitgeteilt und in Praxi mit ihnen geteilt.

\section{Zukunft Medizin Schweiz}

Die Schweizerische Akademie der Medizinischen Wissenschaften (SAMW) engagiert sich seit mehreren Jahren im Projekt «Zukunft Medizin Schweiz» (früher «Neu-Orientierung der Medizin»). Seit Anfang 2002 wird das Projekt von der FMH und den Medizinischen Fakultäten der Schweiz mitgetragen. In einer ersten Phase soll eine Experten/-innengruppe unter Leitung von Prof. Dieter Bürgin aus Basel die Ziele und Grenzen der Medizin für die Schweiz formulieren.

In den nächsten Monaten werden sich Mitglieder der Experten/-innengruppe in loser Folge in dieser «Kolumne» zu Wort melden und von der Arbeit bzw. den Diskussionen in der Gruppe sowie von ihren eigenen Erfahrungen und Überlegungen berichten.

\section{La médecine en Suisse demain}

Depuis quelques années, l'Académie Suisse des Sciences Médicales (ASSM) s'engage pour le projet «L'avenir de la médecine en Suisse» (initialement appelé «Nouvelle orientation de la médecine»). Depuis l'année dernière, le projet est également soutenu par la FMH et les facultés de médecine suisses. Dans une première phase, un groupe d'expert(e)s sous la direction du Prof. Dieter Bürgin de Bâle définira les buts et les limites de la médecine en Suisse.

Dans les prochains mois, les membres du groupe d'expert(e)s s'exprimeront de temps à autre dans cette rubrique et feront part de leur travail, de leurs discussions, de leurs expériences ainsi que de leurs réflexions. 
Unsicherheit ist Teil des Lebens. Sie ist deshalb auch ein natürlicher Teil der Medizin und müsste im Prinzip in der Arzt-Patient-Beziehung verarbeitet und von beiden Partnern getragen werden können. Das ist heute nicht mehr so klar und so einfach, wie es sein müsste. In der Schweiz werden im Gesundheitswesen weit mehr als 40 Milliarden Franken pro Jahr umgesetzt. Deshalb interessieren sich immer mehr Menschen und Organisationen dafür, am Wirtschaftsfaktor Medizin zu partizipieren und für sich einen Gewinn herauszuholen. Dies hat dazu geführt, dass die Arzt-Patient-Beziehung schon seit einiger Zeit nicht mehr nur dem Ziel einer optimalen Versorgung der Patientinnen genügen muss, sondern noch vielen anderen Ansprüchen. So üben z.B. die Politiker, die Krankenkassen und die Verwaltungen der Spitäler einen starken Spardruck aus. Auf der anderen Seite preisen die industriellen Betriebe ihre Innovationen an, die zu einer besseren Versorgung der Patientinnen beitragen sollen. Zudem sind die Patientinnen heute autonomer geworden und stellen Forderungen. Auch die Ärzte selber sind gegenüber wirtschaftlichen Anreizen nicht immun. Somit bewegt sich heute die Arzt-Patient-Beziehung in verschiedenen Spannungsfeldern, die nichts mehr mit ihrem primären Ziel zu tun haben. Die verschiedenen Einflussnahmen auf die Medizin sind aber deshalb besonders wirksam, weil sie sich die Unsicherheit medizinischer Entscheidungen zu Nutze machen. Gerade, weil alles auch anders entschieden werden kann, soll die Medizin dem Spardruck genügen oder sollen die Ärzte dieses oder jenes Produkt wählen. Wer sind in dieser komplexen Situation die Gewinner und die Verlierer?

Weder die Prämienzahlerinnen noch die Patienten gehören zu den Gewinnern. Die Prämien sind trotz aller Bemühungen zur Kostendämpfung jedes Jahr kräftig weiter angestiegen. Der Spardruck auf die Leistungserbringer hat aber die Zeitdauer der Kommunikation in der ArztPatient-Beziehung und in der Pflege-Patient-Beziehung minimisiert und dafür den Aufwand in der Administration vergrössert. Zudem hat der Spar- und Leistungsdruck heute ein Ausmass angenommen, welches die Attraktivität des Arzt- und des Pflegeberufs gefährdet. In Deutschland z. B. beginnt ein grosser Teil der Absolventen des Medizinstudiums keine Weiterbildung in einem Spital. Es ist plötzlich ein Mangel an Assistenzärztinnen eingetreten, der sich in einigen Jahren bei den erfahrenen Ärzten bemerkbar machen wird. Der Mangel an Nachwuchs bei den Pflegeberufen ist heute schon evident. Somit können wir feststellen, dass die Ärztinnen und die Pflegenden unzweifelhaft bei den Verlierern sind. Den Politikern, den Krankenkassen und den Verwaltungen der Spitäler sind bisher keine Verluste entstanden. Sie haben vielleicht etwas an Macht gewonnen, werden aber längerfristig auch unter immer grösseren Druck geraten, wenn infolge eines Ärzte- und Pflegemangels die medizinische Versorgung leiden wird. Bleibt noch die Industrie, welche die Medizin mit immer neuen Produkten ausrüstet. Bisher hat sie dank Innovation immer wieder wachsen können und damit zur Verteuerung der Medizin beigetragen. Es wird aber der Tag kommen, an dem das Gesundheitswesen nicht mehr alle neuen Produkte wird finanzieren wollen. Auch hier ist ein Ende des Booms abzusehen.

Die Expertengruppe «Zukunft Medizin Schweiz» wird sich mit der Frage auseinanderzusetzen haben, wie aus einer Verlierer-VerliererSituation eine Win-Win-Situation entstehen kann. Meines Erachtens müssen sich in erster Linie die Angehörigen der Gesundheitsberufe darüber klar werden, welches der zentrale Auftrag der Medizin ist und welche Bedingungen erfüllt sein müssen, damit sie ihm gerecht werden können. Diesen Auftrag und diese Bedingungen müssen sie dann gegen jedes Anspruchsverhalten von innen und von aussen verteidigen. Nur so sind die Arzt-Patient-Beziehung und die Pflege-Patient-Beziehung wieder ihren Bestimmungen zuzuführen und von fremden Einflüssen zu befreien. Die Unsicherheit medizinischer Entscheidungen darf nicht zu Manipulationen Anlass bieten, sondern muss Teil der Programme zur Verbesserung der Medizin bleiben. Auf dieser Basis wird die Aufgabe der Ärzte und der Pflegenden wieder sinnstiftend und befriedigend, und nur so ist den Patienten optimal zu helfen.

Bisher in der Rubrik «Zukunft Medizin Schweiz» erschienen:

Bürgin D. Wohin steuert die Medizin? Schweiz Ärztezeitung 2003;84(13):578-9.

von Castelberg B. Ist die Zukunft der Medizin weiblich? Schweiz Ärztezeitung 2003;84(20):1059-60.

Amstad H. Von «Ärzten» und «guten Ärzten». Schweiz Ärztezeitung 2003;84(43):2250-51. 